\title{
TERAPIA DE PAREJA: IMPORTANCIA DEL CONTRATO PARA EL CAMBIO
} Couple therapy: Importance of contract for change

\section{Resumen}

El Análisis Transaccional es una teoría de la psicología creada por el Dr. Eric Berne. Se denomina transaccional porque hace énfasis en el estudio y análisis del intercambio en la comunicación entre dos o más personas, como se da en la terapia de pareja. Todas las personas sanas podemos "redecidir" cambios favorables mediante un trabajo de crecimiento personal. El AT posee técnicas psicoterapéuticas que posibilitan este cambio, siendo importante para ello el establecer un contrato para este cambio.

Palabras claves: Insight, contrato, cambio.

\begin{abstract}
The Transactional Analysis is a theory of Psychology created by Dr. Eric Berne. It is called transactional because it emphasizes the study and analysis of communication exchange among two or more individuals, as given in couple therapy. All healthy people can "redecided" favorable changes through a personal growth work. The TA has psychotherapeutic techniques that make possible this change, which is important for it in order to establish a contract for this change.
\end{abstract}

Keywords: insight, contract, change.

\footnotetext{
* Docente Universidad Femenina del Sagrado Corazón. Director de Animus. juliosilvac@ hotmail.com
} 
El objetivo en el presente trabajo es el de presentar una perspectiva sobre las formas en las que el Análisis Transaccional es utilizado para promover el cambio. Quiero trasmitir a través de este artículo la importancia de las relaciones de pareja pues resultan ser un excelente ejemplo de lo que es el entendimiento de las personas en sus roles de pareja. Y de cómo el AT resulta ser un modelo de terapia idónea para este tipo de intervención.

A partir de mi experiencia en el trabajo con parejas en general, casadas, convivientes, de novios o enamorados, etc. Encuentro que esto puede ser como un transitar sobre un terreno movedizo. Las relaciones de pareja son complejas; pues atraviesan por diversas etapas: por lo general comienzan con una especie de ceguera romántica pues la elección se hace desde el estado del yo Niño y pasan por periodos más o menos difíciles de adaptación a las diferencias reales del modo de ser de cada persona, a los cambios en el modo de vida y a las diversas necesidades mutuas. Las relaciones de pareja tienen inexplicables maneras de convertirse en lo que uno menos hubiera esperado o deseado. En ocasiones incluso en la réplica de la relación con uno de los padres o progenitores. Con cierta frecuencia se dice que sus miembros tienden a complementarse para compensar sus respectivas flaquezas, pero ese equilibrio complementario, así como puede enriquecer una relación, es capaz de provocar una situación conflictiva. A pesar de ello sean cuales fueran las circunstancias, la mayoría de los seres humanos prefiere vivir en pareja a tener que vivir solos.

La importancia de los vínculos en la relación de pareja y en cómo éstas interactúan son un poderoso recurso para la terapia en el AT. Una pregunta como ¿Qué necesita cada uno de ustedes del otro para sentirse un poco menos (molesto, afectado, temeroso, enojado)? Puede convertir el "resentimiento" en "afecto". Las relaciones de pareja tienen que ver justamente con aquello que en el AT llamamos CARICIAS. Que dicho sea como lo señala Claude Steiner (1980) es uno de los instrumentos más valiosos con los que se cuenta en la terapia de parejas en el AT.

\section{¿POR QUÉ USAR CONTRATOS?}

Lo primero y más importante, en el énfasis sobre los contratos en la práctica del AT se basa en el supuesto filosófico de que "la gente está bien" en general como lo señal Thomas Harris (1985). El terapeuta y las parejas se relacionan entre ellos como iguales. Por lo tanto, comparten responsabilidades con respecto al cambio que la pareja quiere realizar.

Esto se deriva de la creencia de que todo el mundo es capaz de pensar y es, en última instancia, responsable de su propia vida. La cual vivirá con las consecuencias de lo que decida. Por consiguiente, está en manos de la pareja, no del terapeuta, el decidir qué quieren para ellos en sus vidas. El trabajo del terapeuta es indicar todo lo que parezca disfuncional.

Para que esta responsabilidad compartida tenga sentido, ambas partes necesitan tener clara la naturaleza del cambio que se desea y la contribución que cada uno realizará hacia su consecución.

La pareja que a continuación describo las llamaremos JUANA y SIMÓN ambos describen su relación desde diferentes puntos de vista y están preocupados porque encuentran que su relación ha tendido a desgastarse. Están casados desde hace 5 años, no tienen hijos, vienen a propuesta de SIMÓN. Ella tiene 32 y él 33 años. Han hecho varios intentos por arreglar sus diferencias y encuentran que no lo logran por ellos mismos. Ambos vienen motivados a encontrar una solución a lo que está sucediendo:

JUANA: con SIMÓN ya no estamos como antes, y él no quiere pasar más tiempo conmigo. No me logro explicar qué pasa con nuestra relación. Él antes me hablaba en casa de muchas cosas y me preguntaba qué pensaba. Ahora ya no lo hace.

SIMÓN: esto no es culpa mía. Desde que JUANA empezó a trabajar esos horarios extras, eso es lo que más le importa. Ya no tiene tiempo para nosotros. (al terapeuta).

TERAPEUTA: díselo a JUANA, dirígete a ella...

JUANA: no es cierto. Yo trato de lograr que hables, pero todo lo que haces es estar ahí sentado viendo la televisión todo el día. (al terapeuta) ¿Qué haría usted si su esposo la ignora? 
SIMÓN: no es que la ignore. No tiene sentido hablar con ella porque le encanta discutir por cualquier cosa y nada de lo que yo digo o haga o deje de decir o deje de hacer le parece bien. (al terapeuta). ¿Qué está mal en mí?

Aparentemente el terapeuta no podría contestar ninguna de las preguntas que le han dirigido sin tomar partido. Además, si diera una respuesta al contenido de esas quejas, probablemente provocaría que escalen más cólera cada uno y se repliquen mutuamente. No obstante, pudo buscar algún elemento común en el motivo de las quejas de ambos y utilizarlo como recurso para interactuar mejor.

TERAPEUTA: ustedes dos parecen no estar satisfechos con la falta de atención mutuamente. Los dos parecen querer lo mismo. ¿En algún momento se sintieron los dos satisfechos por la atención uno del otro?

JUANA y SIMÓN: afirmativamente lo expresan con la cabeza.

Estas preguntas merecieron la atención de JUANA y SIMÓN hacia el hecho de que seguía existiendo una relación entre ellos, aun cuando quizá se sintieran muy alejados. Un pequeño cambio puede llevar a cambios más grandes. Si JUANA y SIMÓN hubieran redefinido su problema como "no pasamos suficiente tiempo juntos" e ideado una solución satisfactoria para ambos, parecería que la recomendación sería poner fin a la terapia. De acuerdo con mi experiencia, esta especie de insight o comprensión suele darse solo excepcionalmente. En este caso, el esfuerzo podría consistir en aclarar con la pareja por qué razón el hecho de pasar más tiempo juntos representa una mejora para ellos. Es posible que sus respuestas se refieran al aspecto emocional y a un sentimiento de mayor acercamiento o reconocimiento. Esta aclaración fortalecería su insight de la relación.

TERAPEUTA: visto esto entonces el contrato por ahora será como hacer para compartir más tiempo juntos.

En lo fundamental, el trabajo con parejas desde el AT no difiere mucho del que se realiza individualmente. Sin embargo, la práctica es más difícil porque la solución tiene que satisfacer a las parejas en cuestión, y esta se compone de personas con puntos de vista diferentes. Para superar este obstáculo, es necesario que ambos miembros de la pareja confíen en que el terapeuta no se pondrá en contra de ninguno de ellos. La tarea de transmitir aceptación y comprensión a dos personas que, cada una por su lado, creen estar en lo cierto es difícil, incluso, para el más experimentado de los terapeutas.

Los pasos que se describen a continuación fueron desarrollados para mostrar cómo pueden manejarse estas cuestiones complejas con un mayor grado de confianza. El primer paso consiste en determinar si la terapia de pareja según el AT es apropiada para una pareja en particular.

\section{LA SESIÓN CONJUNTA DE EVALUACIÓN}

La primera sesión en terapia de parejas, según el AT, debe ser conjunta porque de este modo el terapeuta tendrá una muestra de cómo se relacionan entre ellos. También podrá apreciar su deseo y su capacidad de trabajar en la relación. Solo es posible hallar una solución si hay personas que lo desean. Es improbable que el trabajo con parejas sea productivo cuando las partes no tienen metas similares y no se esfuerzan por contribuir a una solución. Así, un esposo dispuesto a salvar el matrimonio a toda costa, y una esposa que no sabe con certeza si desea seguir casada no está preparado para iniciar una terapia de pareja según el AT.

Una excepción importante la constituyen los clientes que no desean estar en terapia y solo asisten para complacer a otra persona. En una ocasión en que asistí a una pareja de esposos, el esposo manifestó que él venia porque ella le había insistido. El dialogo fue más o menos así:

PACIENTE: la verdad que yo vengo por ella.

TERAPEUTA: ¿cómo fue que su esposa le dijo para venir?

PACIENTE: ella me dijo vamos.

TERAPEUTA: (interrumpí) y Ud. acepto.

PACIENTE: perplejo y en silencio. 
TERAPEUTA: se da cuenta que responsabiliza a su esposa por decisiones que usted libremente decide.

En una situación como esa, lo mejor es conversar con los clientes sobre las diferencias en una sesión aparte y aclarar sus necesidades. En ocasiones, un cónyuge indeciso necesita antes trabajar individualmente para tomar una decisión. Si vemos que uno de los cónyuges indecisos va a necesitar mucho tiempo para adoptar una decisión, es preferible derivar a ambos a un tratamiento individual e invitarlos a retornar para iniciar una terapia de pareja en caso de que hayan resuelto trabajar para mejorar su relación o darla por concluida.

En la primera sesión de terapia de una pareja conviene decir a los clientes que el contrato terapéutico concierne a su relación y no a ellos individualmente, y que la meta será negociar sus diferencias que lleve a una solución para ambos. De ese modo se aclara que el terapeuta no tomara partido. Incluso opino que las parejas desde su ADULTO pueden ser capaces de tomar acuerdos en ocasiones incluso de sus propios desacuerdos.

Comenzaremos examinando una de las características centrales en la práctica del AT: la utilización de contratos.

Berne (1991) definió el contrato como un compromiso explicito bilateral hacia un curso de acción bien definido. En la definición de James y Jongeward: (1997) "un contrato es un compromiso adulto con uno mismo y/o alguien más para llevar a cabo un cambio."

Un contrato es una meta objetiva de cambio positivo de conducta. Debe formalizarse entre los A, del cliente y del facilitador. Hacer un contrato A-A es uno de los procedimientos más importantes para reforzar el estado A del yo, ya que formalizar un contrato es crear un compromiso del A consigo mismo y/o con alguien más para efectuar un cambio.

El objetivo final ha de ser específico, observable, de modo que el terapeuta y el cliente, lo mismo que otras personas, puedan saber cuándo ha sido logrado. Los contratos son una decisión y una actuación para apartarse de los aspectos negativos de la propia conducta y establecer nuevos aspectos positivos. El contrato debe ser claro, conciso y directo. Los elementos de los que debe constar son:

- Reconocimiento de que se tiene un problema de conducta.

- La decisión de trabajar en ese problema específico y observable de conducta.

- Una declaración sobre la meta específica objetivo de conducta- que se espera alcanzar, expresada en un lenguaje lo suficientemente simple para que lo comprenda el $\mathrm{N}$.

- Las probabilidades de alcanzar la meta deben ser realizables.

\section{ESPECIFICIDAD Y CONDICIONES DE LOS CONTRATOS}

En el AT distinguimos dos tipos de contrato: el contrato comercial o de negocios (acuerdo comercial) y el clínico o contrato de tratamiento.

En el contrato de tratamiento, la pareja establece con claridad los cambios que quieren llevar a cabo y especifican que están dispuestos a hacer para ayudar a ocasionar estos cambios. El terapeuta dice si está dispuesto a trabajar con la pareja en la consecución de los cambios que sea y expone cual será su aporte al proceso.

- Quiénes forman las dos partes.

- Qué es lo que van a hacer juntas.

- Cuánto tiempo les llevará.

- Cuál será el objetivo o el resultado de este proceso.

- Cómo sabrán cuándo han llegado al objetivo.

- Cómo beneficiará a su relación.

Se referirá a un área de conducta en la cual la pareja tiene problemas que no pueden aún resolver solos:

- El cambio de conducta debe formularse en términos positivos.

- El cambio de conducta que se busca debe ser observable y medible. No es recomendable ajustar contratos sobre lo que se piensa o lo que 
se siente, sino sobre lo que se actúa (se hace o dice).

- La redacción será simple y concreta. Debe poder entenderla un niño de 8 años.

- La conducta que se busca debe ser legal y ética.

- El cumplimiento deberá depender de la pareja.

- Siempre que corresponda deberá tener un plazo de cumplimiento.

- Deberá referirse a una conducta específica y concreta.

\section{LOS “CUATRO REQUISITOS” DE STEINER}

Claude Steiner (1980) discípulo de Berne ha expuesto cuatro requisitos para hacer un contrato sólido. Se derivan de la práctica de contratos en ámbitos jurídicos.

1. Consentimiento mutuo.

2. Retribución.

3. Competencia Mutua.

4. Tema legal.

\section{NEGOCIANDO UN CONTRATO}

Un primer paso en mi quehacer con parejas consiste en negociar un contrato terapéutico para alcanzar una meta específica mensurable, comprobable y asequible a partir de la terapia. Por lo común, incluye cambios en el modo de pensar, de sentir y de comportarse, aunque a veces sólo afecta una de estas áreas.

A modo de ejemplo, Bob Goulding, en una entrevista realizada por Michael Hoyt (1995) señala, que el suele preguntar a cada cliente: "¿Qué quiere cambiar hoy con respecto a usted?": Con ello afirma la creencia de que:

1. El cliente es el responsable de su propio cambio;

2. El cambio es posible,

En la negociación del contrato, el terapeuta y la pareja son socios en el sentido de que éstos tienen derecho a conocer la opinión sincera de él.
Una terapia eficaz debe partir de un buen contrato. Aprendí de mi maestro Rafael Junchaya (1989) que un buen terapeuta deberá ayudar a sus pacientes a escoger contratos rápidos y viables. En palabras de él, este contrato tiene que ver con hacer un contrato para aclarar que es lo que la persona espera de la terapia y que es lo que quiere cambiar.

Cuando una pareja dice que quieren "conocerse mejor a sí mismos" o "averiguar por qué ellos...", no habla de un cambio personal. El terapeuta tiene que preguntarle: “¿En qué áreas específicas de su vida quieren conocerse mejor?", ¿Qué cambios personales desea producir una vez que se conozca mejor y haya averiguado el por qué de su conducta?". Si las parejas responden: "Verá usted, nos gustaría digamos... vivir más o menos asî", es responsabilidad del terapeuta enseñarle a establecer metas de corto plazo que sean pequeñas, diferenciadas, claras y comprobables.

Durante el proceso de fijación del contrato, las personas aprenden a pensar en forma creativa acerca de su vida actual: "¿Cómo es exactamente mi vida? ¿Qué me agrada y qué me desagrada en ella? “¿En qué forma precisa queremos cambiar para enriquecer nuestra vida actual?". Esto incluye salud, trabajo, amor y trato social.

Esta exploración del presente puede continuar entre sesiones. El terapeuta quizá sugiera tareas específicas, por ejemplo: "Me ha hablado con claridad de su relación con su hijo, pero no ha sido tan clara al referirse a lo que cuando usted y su marido empiezan a discutir. Escúchese y escúchelo por unos días y, si quiere, tome apuntes. Establezca con exactitud cómo terminan".

Mientras establece su contrato, las parejas también aprenden a pensar en su futuro: “Cómo queremos ser mañana, el año próximo, dentro de dos años?". Se pregunta. Un plan de acción fácil consiste en pedirle que finja haber terminado en ese momento la terapia con un éxito arrollador: ha logrado todos los cambios personales que podía desear. Luego, preguntémosle: "¿Dónde vive? ¿Con quién? ¿Qué hace en su nueva y exitosa vida? ¿En qué han cambiado sus pensamientos, sentimientos y conducta, con respecto a los que tenía al iniciar la terapia?". 
Desde luego, mientras exploran el presente y el futuro junto con las parejas, pueden ventilar cualquier problema de transferencia que sea importante resolver: "Cuando los interrogo sobre el futuro, ustedes me hablan del pasado. Cuando le pido que alce la voz, la bajan. Sospecho que una de sus formas de tratar entre ustedes es hacer lo contrario de lo que esperan".

\section{CONTRATOS Y LA AGENDA ENCUBIERTA}

En cualquier relación, las partes pueden intercambiar mensajes ulteriores. Es probable que esto suceda en situaciones en las que se busca cambio personal, ya que estos cambios generalmente significan un reto al marco de referencia de las personas. Es probable que el terapeuta y la pareja entren en su relación de trabajo con una agenda encubierta además de su agenda a nivel social. Una función importante del contrato es la de explicar la agenda encubierta. Poniendo de manifiesto mensajes ulteriores, el hacer un contrato claro corta los juegos psicológicos y ayuda tanto a la pareja como al terapeuta a mantenerse fuera del triángulo dramático.

El terapeuta tiene su propio marco de referencia y será distinto del que tiene la pareja. Luego éste entrará en su relación llevando sus propias definiciones internas sobre los tipos de cambio que son "buenos" para la gente. Sin contrato, sería tentador para el psicólogo asumir que las definiciones de sus clientes fueran las mismas que las suyas propias. Puede hacer suposiciones sobre los "verdaderos" objetivos que la pareja ha de conseguir.

En esta situación, es probable que el terapeuta entre en un rol del triángulo dramático. Puede que comience "llevando por su propio camino" al cliente en una dirección en particular, actuando de este modo como perseguidor.

De forma alternativa, el psicólogo puede pensar: "esta pareja necesita realizar este u otro cambio. No lo han hecho aún. Por lo tanto, están en una situación difícil y no se las puede arreglar sin mi ayuda”. De esta manera, pasaría al rol de salvador.

Es probable también que el cliente tenga tanto una agenda encubierta como una manifiesta. Llegando al psicólogo, ha declarado a nivel social que hay algún cambio que quiere hacer. En este caso, pasará mensajes ulteriores al psicólogo como: "he venido a cambiar, pero soy incapaz de hacerlo" o "he venido a cambiar, pero tú no me puedes hacer cambiar".

Si la agenda encubierta sigue hacia delante por ambas partes, el terapeuta y el cliente adoptan roles complementarios en el triángulo dramático, abriendo el camino para el chantaje emocional y los juegos.

Una función del contrato es la de anticiparse a esto. Por medio de la negociación de objetivos claros y métodos de cambio. Este proceso ayuda a traer la agenda encubierta a la conciencia Adulto, para que de esta manera ambas partes puedan contrastarla con la realidad. En cambio el contrato puede tener que ser revisado y si es necesario, renegociado muchas veces durante el proceso de cambio.

\section{CONTRATOS Y ORIENTACIÓN DE OBJETIVO}

La mayoría de las parejas llegan a consulta trayendo un problema que quieren tratar de resolver. Uno de los propósitos de hacer un contrato es el de quitar el foco de atención del problema y cambiarlo por el objetivo de cambio. Roberto Kertész (1997).

En el proceso de hacer un contrato, ambos, la pareja y el terapeuta, deben construir necesariamente una imagen mental del resultado que desean de su trabajo juntos. Cuando se orientan ellos mismos de esta manera hacia un objetivo claro, automáticamente movilizan los recursos personales que necesitan para conseguir ese resultado.

En contraste, si el terapeuta y la pareja atienden fundamentalmente al "problema", habrán tenido que construir una imagen mental de ese problema. Sin querer hacerlo, estarán realizando visualizaciones negativas, dirigiendo sus recursos a examinar el problema en vez de resolverlo.

Otra ventaja al establecer un objetivo de contrato claramente estipulado: ofrece a ambas partes una manera de saber cuando han completado sus trabajos juntos. También les permite evaluar los progresos que están realizando por el camino. De esta forma, el uso de 
contratos previene la situación en la que el tratamiento puede durar para siempre, de manera interminable, con la pareja y el terapeuta invirtiendo meses y años "trabajando sobre" los problemas de la pareja.

\section{ESTABLECIENDO UN CONTRATO EFECTIVO}

A modo de resumen presento aquí las características principales que buscamos los terapeutas del AT en un contrato efectivo. Como indicaron James y Jongeward, (1997) podemos hacer un contrato para el cambio tanto con nosotros mismos como con un terapeuta.

Por ejemplo el ejercicio que aparece a continuación está basado en un procedimiento de establecimiento de contrato:

- Decídanse por un cambio personal que quieran llevar a cabo. Anótenlo usando cualquiera de las palabras que les vengan a la cabeza.

Un objetivo de contrato debe de ser formulando en palabras positivas. A menudo, la redacción inicial de un objetivo contendrá negativas. Por ejemplo, la persona puede que quiera dejar de fumar o controlar la bebida, perder peso, o no estar asustada de las figuras de autoridad. A largo plazo, estos "contratos de dejar" y "contratos no" nunca funcionan. En parte, esto es debido a la manera en la cual el objetivo del contrato actúa como visualización. No se puede visualizar "algo no". (Si dudas sobre esto, visualiza "no un elefante rojo") cuando intentamos hacerlo, automáticamente aparece una imagen mental de cualquier cosa que siga al "no" o cualquier otra palabra negativa. Por ejemplo, si una persona acepta el reto de "dejar de fumar", no puede encarar ese objetivo sin visualizar continuamente la actividad problema de la cual estásaliendo para dejarlo.

Existe una buena razón en la teoría de AT para la inefectividad de los "contratos dejar". Recordemos que toda conducta de guion representa la mejor estrategia de niño para sobrevivir, consiguiendo caricias y cubriendo necesidades. Entonces, ¿qué sucede si simplemente se contrata el "dejar de hacer" es conducta de guión? Por lo menos, se ha fallado en darle a su si mismo en Niño cualquier directiva clara sobre que es lo que va a hacer, simplemente se añade uno más a la interminable lista de "no hagas" y "deja de" que se grabó de sus padres cuando era joven. O lo peor, puede que esté contratando el abandonar una conducta que en Niño ha estado percibiendo como esencial para su supervivencia.

Para lograr un contrato efectivo, se debe especificar el positivo que le proporcionara en Niño con una directiva clara para la acción. Debe proporcionar una nueva opción de supervivencia para cubrir las necesidades que sea, al menos, igual de bueno que la opción de guión.

$\mathrm{Si}$ el deseo comunicado contiene palabras negativas, vuelva a formular la frase para que contenga solamente positivos. La declaración con la nueva formulación, dirá que positivo se va a utilizar para sustituir el negativo.

El contrato debe establecerse para una meta alcanzable, teniendo en cuenta la situación y los recursos actuales. En general, consideramos "alcanzable" cualquier cosa que es físicamente posible. Observemos que esta condición implica que solamente se puede contratar un cambio que deseas realizar en ti mismo. No es físicamente posible "hacer" que algún otro cambie.

- Comprobarque el cambioque se desea es posible para uno. Como propuesta de comprobación, pregúntese: ¿lo ha logrado alguna otra persona del mundo? En caso afirmativo, debe ser incluida como posible.

La meta debe ser específica y observable. Tanto uno como los demás deben poder ver claramente si se ha logrado la meta. Tener cuidado con las metas sobregeneralizadas y con las comparativas. A menudo, la gente comenzara con metas globales como: "quiero ser una persona cálida y extrovertida" o "quiero acercarme a los demás". Realizar un contrato de este tipo es involucrarse en un trabajo permanente, puesto que las metas declaradas no son lo suficientemente específicas como para que cualquier persona sepa si se han logrado.

- ¿Sabrá uno y los demás si se ha logrado el cambio deseado? Declarar esta respuesta con 
todos los detalles de lo que uno y los demás podrán ver y oír lo que se hace diferente. Si la meta está relacionada con la forma en la que se relaciona con los demás, específicamente que personas, por su nombre.

El cambio que se está intentando lograr debe ser seguro. Utilizar la valoración de adultos y tener en cuenta tanto la seguridad física como la adecuación social.

- ¿Es el cambio que se desea seguro?

El objetivo del contrato debe decidirse desde el Adulto, con colaboración del Niño Libre. En otras palabras, debe ser adecuado a la situación de Adulto y sus habilidades y ayudar a satisfacer las necesidades autenticas del Niño en lugar de negarlas. Un contrato establecido desde el Niño Adaptado tiene el efecto de profundizar el guion.

- Comprobar: ¿cuánto deseo este cambio para mí, más que para complacer a otros, obtener la aprobación de alguien o rebelarme contra alguien? Los "demás" o "alguien" pueden ser personas de mi pasado o presente. Otra forma de realizar esta pregunta de comprobación es: “¿Qué significa el cambio para mi?”.

Para lograr la meta, se necesita movilizar los recursos del Niño junto con los del Adulto y el Padre. Por tanto las metas del contrato AT se formulan en "lenguaje de niño de ocho años", en palabras comprensibles a la parte Niño.

- ¿Se ha formulado la meta en palabras que comprendería un niño de ocho años inteligente? En caso contrario, vuelve a formularla para que así sea. Lograr la meta tendrá algún costo. En términos de tiempo, dinero, compromiso, intranquilidad, despedidas o enfrentarse al miedo al cambio.

- Comprobar: ¿qué costara lograr este cambio? Ahora que se ha calculado el costo, ¿aún desea el cambio?

Los pasos a continuación suponen un compromiso con una acción específica:
- Anote al menos cinco cosas que necesita hacer para lograr la meta. De nuevo. Debe ser específico al declarar las acciones tú y los demás podrán ver y oírte realizar. Si estas acciones involucran a personas, especifícalas por su nombre.

- Ahora, de la lista de cosas que necesita hacer, selecciona y anota las que hará en la próxima semana. Anota: "las personas que me apoyarán en este cambio son....". Y añade sus nombres.

\section{UN EJEMPLO DE CÓMO HACER UN CONTRATO}

A continuación el siguiente caso es el de CECILIA una mujer soltera de 33 años que asiste a consulta porque quiere tomar una decisión con respecto a una relación con su pareja CARLOS. Se conocen desde niños. Sus padres son amigos y hasta hace 10 años vivían en el mismo barrio. Asiste ella sola a la consulta, señala que Carlos vendrá la semana siguiente, él solo. Así lo ha pedido él.

CECILIA: tengo una relación de seis años y, trato de tomar una decisión, si continuar con él o no. O si debo mejor terminar con CARLOS.

TERAPEUTA: hábleme algo más de su relación.

CECILIA: algunas veces siento que él no me da suficiente atención, entonces me pregunto si me quiere. Veo que él prefiere irse con sus amigos. Antes de pasarla conmigo.

TERAPEUTA: dígame concretamente que es lo que no hace. Qué atención en particular quiere de él y que no se la brinda.

CECILIA: ya se lo he dicho. Pienso que tenemos diferentes valores, tal vez...

TERAPEUTA: dígame específicamente qué es lo que quiere y no obtiene.

CECILIA: el no me dice que me quiere. Quisiera que él me dijera que me quiere.

TERAPEUTA: $i$ tu quieres que haga eso?

CECILIA: No, no es así.... 
TERAPEUTA: tu quieres que Carlos te quiera.

CECILIA: Sí, quiero amor. Le doy toda mi atención.

TERAPEUTA: le has preguntado a Carlos que es lo que él quiere.

CECILIA: Carlos siempre me dice que no necesita nada.

TERAPEUTA: Hum. Muy bien te preguntaré qué es lo que quieres. ¿Qué es lo que quieres?

CECILIA: él dice siempre que quiere tiempo para estar con sus amigos jugando al póker en nuestro departamento. No quiero sentirme todo el tiempo encima de él. (Empieza a llorar).

TERAPEUTA: que estás sintiendo en este momento.

CECILIA: (sollozando): Ese es el problema. Siento miedo de que lo pueda saturar a él.

TERAPEUTA: De acuerdo, empieza a entender. Por ahora, aceptarás un contrato para aprender a reconocer lo que tú quieres y entonces estará claro si lo que quieres es seguir con CARLOS o no. ¿De acuerdo?

CECILIA: bueno está bien.

\section{APUNTES PARA UN BUEN CONTRATO}

Interesa en los contratos de la terapia. Cómo cambia la gente, no qué cambian. Qué quieren cambiar. Yal escuchar el contrato también escuchamos, determinadas maneras en que lo van a echar a perder, como las palabras que usan y que llamamos cebos. Así tenemos por ejemplo: "Tratar" "Lo que me gustaría hacer" o "Lo que mi esposa quiere que yo haga" o "Lo que mi esposo quiere que yo haga". Todas las palabras que indican que no van a hacer lo que dijeron que harán. Y los confronto. De modo que al final el contrato está claramente expresado, es funcional y hay poco peligro de que lo sabotee el Niño.

Los pacientes tienen el poder. Lo único que tenemos es este conocimiento de cómo ellos pueden cambiar. Son los que cambiarán - tengámoslo presente en todo momento. Luego repasemos el proceso del trabajo que tiene que hacerse. Y en verdad, no importa qué clase de terapia se hace en términos de si es terapia de Redecisión o algo distinto - cualquiera con buenas habilidades en cualquier clase de terapia que haga logrará el mismo cambio.

En una ocasión asistí a una pareja de novios que convivían desde hace algunos años eventualmente. Tenían unos 36 años aproximadamente no tenían hijos y ella tenía en casa una niña de unos 6 años que era la hija de la empleada. La niña vivía ya con ella y quería adoptarla legalmente y él no. María es asistenta social y Federico es pintor. La sesión se desarrollo más o menos así:

ELLA: bueno venimos porque queremos saber si podríamos ser buenos papás.

TERAPEUTA: qué opina a lo dicho por su pareja.

ÉL: no necesariamente es así como ella lo plantea. En realidad ella quiere hacer el trámite legal para adoptar a una niña. Yo particularmente no deseo lo mismo.

ELLA: pero nosotros habíamos dicho que tendríamos hijos.

ÉL: si pero los nuestros no necesariamente los de otra persona.

TERAPEUTA: ustedes que esperan al venir a terapia.

ELLA: si me gustaría que él aceptara la adopción de Marie.

ÉL: yo quisiera que nos diéramos un tiempo para decidir.

TERAPEUTA: bueno nuestro contrato va a consistir en acordar si están de acuerdo en adoptar a Marie o no.

ELLA: me parece categórico pero estoy de acuerdo.

ÉL: claro creo que es lo primero que necesitamos.

TERAPEUTA: bien entonces: que la lleva a Ud. a pensar en adoptar a Marie.

ELLA: pienso que con ella completaríamos una familia.

EL: pero eso puede ser teniendo nuestros propios hijos.

TERAPEUTA: Ud. quiere ser madre y hacer padre a Federico. 
ELLA: si pero también pienso que debo ayudar a la sociedad adoptando a un niño.

EL: ella trabaja en una ONG y se siente como obligada a tener que asumir a una niña.

TERAPEUTA: entonces queda claro que no solo es por la niña en sí. Si no por lo que esto significa para Ud.

\section{OBJETIVOS DEL CAMBIO EN AT}

Hasta ahora hemos visto como se negocian los objetivos específicos de contrato entre el Terapeuta de AT y el cliente. Pero ¿Qué producto final desean al proceso de cambio? ¿Cómo saben el cliente y el terapeuta cuando han completado su trabajo juntos?

Recordemos que un tratamiento puede concluir cuando se cumple el contrato terapéutico.

Para finalizar este articulo solo mencionare lo que es un objetivo importante del cambio al cual Berne llamó Autonomía, y lo describió como la expresión de tres capacidades: la consciencia, la espontaneidad y la intimidad.

\section{BIBLIOGRAFÍA}

Berne, E. (1981). Análisis transaccional en psicoterapia: una psiquiatría sistemática, individual y social. Buenos Aires; Psique.

Berne, E.(1982). Hacer el Amor. Argentina: Alfa.

Camino, J. y Coca, A. (2009). Juegos, Guion y Pareja. Revista de Análisis Transaccional y Psicología Humanista, 60. Madrid.

Harris, T. (1974) Yo estoy bien - Tu estás Bien. España: Grijalbo

Harris, T. y Harris, A.(1985) Para estar siempre bien. España: Grijalbo.

Fecha de recepción: 12 de agosto 2013

Fecha de aprobación: 14 de noviembre 2013
Hoyt, M. (1995) Contacto, contrato cambio: una conversación con Bob Goulding. Journal de Análisis Transaccional. San Francisco USA

Germaine, J. y Darby, J.(1995) A manera de Introducción: Robert L. Goulding y la Terapia de Redecisión. Journal de Análisis Transaccional. San Francisco USA

James, M.; Jomgeward, D. (1997). Nacidos para triunfar: análisis transaccional con experimentos Gestalt. Bogotá: Fondo Educativo Interamericano

Junchaya, R. (1989). No es nuestro destino es nuestra programación. Volumen I el a,b,c... Lima.

Kertész, R. (1977). Introducción al análisis transaccional: los juegos psicológicos. Buenos Aires: Paidós.

Kertész, R. (1997). Análisis Transaccional Integrado. Buenos Aires: IPPEM.

Masso, F. (2007). Análisis Transaccional I. Cómo nos hacemos persona. Madrid: CCS

Pedreira, A. (2010). Impacto de los Juegos Psicológicos en las distintas etapas de la dinámica amorosa. Ponencia de clausura presentada en el XV Congreso Español de Análisis Transaccional en Zaragoza España. Mayo.

Román,J.M.;Senlle,A.(1994). AnálisisTransaccional: modelo y aplicaciones. Salamanca: Amarú.

Steiner, C. (1980). Libretos en que participamos. México: Diana.

Steiner, C. y Perry, P. (1998). La Educación Emocional. Buenos Aires: Grupo Zeta.

Stewart, I.; Joines, V. (2011). AT Hoy: Una nueva introducción al Análisis Transaccional. Madrid, CCS. 